\title{
ON HIGHER ORDER ANGULAR DERIVATIVES - AN APPLICATION OF FAÀ DI BRUNO'S FORMULA
}

\author{
YIFEI PAN AND MEI WANG
}

\begin{abstract}
We study the singular behavior of $k$ th angular derivatives of analytic functions in the unit disk in the complex plane $\mathbb{C}$ and positive harmonic functions in the unit ball in $\mathbb{R}^{n}$. Faà di Bruno's formula is a crucial tool in our proofs.
\end{abstract}

\section{INTRODUCTION}

In this paper, we study the singular behavior of $k$ th angular derivatives of analytic functions in the unit disk in the complex plane $\mathbb{C}$ and positive harmonic functions in the unit ball in $\mathbb{R}^{n}$. Faà di Bruno's formula plays an important role in our proofs.

Let $\mathbb{D}=\{z:|z|<1\} \subset \mathbb{C}$ and $\mathbb{T}=\partial \mathbb{D}$. Let $\varphi: \mathbb{D} \rightarrow \mathbb{D}$ be analytic and $\zeta \in \mathbb{T}$. $\zeta$ is a fixed point of $\varphi$ if $\lim _{r \rightarrow 1} \varphi(r \zeta)=\zeta$. The angular derivative at $\zeta$ is defined as $\varphi^{\prime}(\zeta)=\lim _{r \rightarrow 1} \varphi^{\prime}(r \zeta)$. It is a consequence of the Julia Lemma [6] that the angular derivative at the fixed point exists and that $\varphi^{\prime}(\zeta) \in(0, \infty]$. When the angular derivative of a fixed point is finite, what could be the limiting behavior of the higher order angular derivatives? We describe an asymptotic property of the higher order derivatives of the fixed point in the following theorem.

Theorem 1.1. Let $\varphi(z): \mathbb{D} \rightarrow \mathbb{D}$ be analytic. Let $\zeta \in \mathbb{T}$ be a fixed point of $\varphi$ with angular derivative $\varphi^{\prime}(\zeta)<\infty$. Then $\forall \ell \geq 2$, the $\ell$-th angular derivative

$$
\varphi^{(\ell)}(r \zeta)=o\left(\frac{1}{(1-r)^{\ell-1}}\right) \quad \text { as } \quad r \rightarrow 1
$$

The above is equivalent to $\lim _{r \rightarrow 1}(1-r)^{\ell-1} \varphi^{(\ell)}(r \zeta)=0$ by the definition of the little o notation.

The order $\ell-1$ in Theorem 1.1 is sharp in the sense illustrated in the following proposition and its proof.

Proposition 1.2. For any $\varepsilon \in(0,1)$ and $\zeta \in \mathbb{T}$, there exists an analytic function $\psi(z): \mathbb{D} \rightarrow \mathbb{D}$ such that $\zeta$ is a fixed point of $\psi, \psi^{\prime}(\zeta)<\infty$, and

$$
\lim _{r \rightarrow 1}(1-r)^{\ell-1-\varepsilon}\left|\psi^{(\ell)}(r \zeta)\right|>0, \quad \forall \ell \geq 2 .
$$


Furthermore, for any integer $m \geq 1$, there exists an analytic function $\psi(z): \mathbb{D} \rightarrow$ $\mathbb{D}$ such that $\zeta$ is a fixed point of $\psi$,

$$
\begin{aligned}
& \psi^{(m-j)}(\zeta)<\infty, \quad \forall j \geq 0, \\
& \psi^{(m+k)}(\zeta)=\infty, \quad \forall k \geq 1, \\
& \lim _{r \rightarrow 1}(1-r)^{m+k-1} \psi^{(n+k)}(r \zeta)=0, \\
& \lim _{r \rightarrow 1}(1-r)^{m+k-1-\varepsilon}\left|\psi^{(m+k)}(r \zeta)\right|>0 .
\end{aligned}
$$

Results analogous to Theorem 1.1 can be obtained for positive harmonic functions, as stated in the following theorem.

Theorem 1.3. Let $u$ be a positive harmonic function in the unit ball $B^{n} \subset \mathbb{R}^{n}, n \geq$ 2. Let $\zeta \in S^{n-1}=\partial B^{n}$. Then for $k \geq 1$,

$$
\lim _{r \rightarrow 1}\left\{(1-r)^{n+k-1} \frac{d^{k}}{d r^{k}} u(r \zeta)\right\}=2 \frac{(n+k-2) !}{(n-2) !} \lim _{r \rightarrow 1} \frac{(1-r)^{n-1}}{1+r} u(r \zeta) .
$$

Consequently,

$$
\lim _{r \rightarrow 1}\left\{(1-r)^{n+k-1} \frac{d^{k}}{d r^{k}} u(r \zeta)\right\}=0
$$

except possibly on a countable set of points on the sphere.

From the proof of Theorem 1.3 we can see that the results can be extended to harmonic functions defined by complex measures. We may restate Theorem 1.3 as the following.

Theorem 1.3'. Let $u$ be a harmonic function in the unit ball $B^{n}, n \geq 2$ defined by a complex measure $\mu$ on $S^{n-1}$ (with the Poisson kernel). Let $\zeta \in S^{n-1}$. Then for $k \geq 1$,

$$
\lim _{r \rightarrow 1}\left\{(1-r)^{n+k-1} \frac{d^{k}}{d r^{k}} u(r \zeta)\right\}=2 \frac{(n+k-2) !}{(n-2) !} \lim _{r \rightarrow 1} \frac{(1-r)^{n-1}}{1+r} u(r \zeta) .
$$

\section{Proof of Theorem 1.1}

First we prove a lemma needed for the proof of Theorem 1.1.

Lemma 2.1. Let $f(z)$ be analytic and $\operatorname{Re} f(z)>0$ for $z \in \mathbb{D}$. Let $\zeta \in \mathbb{T}$. Then

$$
\lim _{r \rightarrow 1}(1-r)^{k+1} f^{(k)}(r \zeta)=\bar{\zeta}^{k} 2 k ! \lim _{r \rightarrow 1} \frac{1-r}{1+r} f(r \zeta) .
$$


Proof. The proof follows the steps similar to the proof of Theorem 1.3 in [2]. First consider the case $f(0)=1$. Since $\operatorname{Re} f(z)>0$, there exists a unique positive Borel measure $\mu$ such that (ref. [3])

$$
f(z)=\int_{\mathbb{T}} \frac{1+\eta z}{1-\eta z} d \mu(\eta), \quad \mu(\mathbb{T})=1 .
$$

Direct calculation yields

$$
f^{(k)}(z)=2 k ! \int_{\mathbb{T}} \frac{\eta^{k}}{(1-\eta z)^{k+1}} d \mu(\eta), \quad k \geq 1 .
$$

Consider $z=r \zeta$. Since

$$
\lim _{r \rightarrow 1} \frac{1-r}{1-r \zeta \eta}= \begin{cases}1, & \eta=\bar{\zeta} \\ 0, & \eta \neq \bar{\zeta}\end{cases}
$$

we have

$$
\int_{\mathbb{T}} \lim _{r \rightarrow 1} \frac{1-r}{1+r} \frac{1+r \zeta \eta}{1-r \zeta \eta} d \mu(\eta)=\mu(\{\bar{\zeta}\}), \quad \int_{\mathbb{T}} \lim _{r \rightarrow 1} \frac{\eta^{k}(1-r)^{k+1}}{(1-r \zeta \eta)^{k+1}} d \mu(\eta)=\bar{\zeta}^{k} \mu(\{\bar{\zeta}\}), \quad k \geq 1 .
$$

By the Lebesgue's dominated convergence theorem,

$$
\begin{aligned}
\lim _{r \rightarrow 1}\left\{\frac{1-r}{1+r} f(r \zeta)\right\} & =\lim _{r \rightarrow 1}\left\{\frac{1-r}{1+r} \int_{\mathbb{T}} \frac{1+r \zeta \eta}{1-r \zeta \eta} d \mu(\eta)\right\} \\
& =\int_{\mathbb{T}} \lim _{r \rightarrow 1}\left\{\frac{1-r}{1+r} \frac{1+r \zeta \eta}{1-r \zeta \eta}\right\} d \mu(\eta)=\mu(\{\bar{\zeta}\}),
\end{aligned}
$$

and

$$
\begin{aligned}
\lim _{r \rightarrow 1}\left\{(1-r)^{k+1} f^{(k)}(r \zeta)\right\} & =\lim _{r \rightarrow 1}\left\{(1-r)^{k+1} 2 k ! \int_{\mathbb{T}} \frac{\eta^{k}}{(1-\eta z)^{k+1}} d \mu(\eta)\right\} \\
& =2 k ! \int_{\mathbb{T}} \lim _{r \rightarrow 1} \frac{\eta^{k}(1-r)^{k+1}}{(1-r \zeta \eta)^{k+1}} d \mu(\eta)=\bar{\zeta}^{k} \mu(\{\bar{\zeta}\}), \quad k \geq 1 .
\end{aligned}
$$

Therefore,

$$
\lim _{r \rightarrow 1}(1-r)^{k+1} f^{(k)}(r \zeta)=\bar{\zeta}^{k} 2 k ! \lim _{r \rightarrow 1} \frac{1-r}{1+r} f(r \zeta) .
$$

If $f(0) \neq 1$, consider

$$
g(z)=\frac{f(z)-i \operatorname{Im} f(0)}{\operatorname{Re} f(0)}
$$

we have

$$
g^{(k)}(z)=\frac{f^{(k)}(z)}{\operatorname{Re} f(0)}, \quad g(0)=1, \quad \operatorname{Re}(g(z))=\frac{\operatorname{Re} f(z)}{\operatorname{Re} f(0)}>0 \quad \text { for } \quad z \in \mathbb{D} .
$$

Thus

$$
\begin{aligned}
\lim _{r \rightarrow 1}(1-r)^{k+1} \frac{f^{(k)}(r \zeta)}{\operatorname{Re} f(0)} & =\bar{\zeta}^{k} 2 k ! \lim _{r \rightarrow 1}\left\{\frac{1-r}{1+r} \frac{f(r \zeta)-i \operatorname{Im} f(0)}{\operatorname{Re} f(0)}\right\} \\
& =\bar{\zeta}^{k} 2 k ! \lim _{r \rightarrow 1}\left\{\frac{1-r}{1+r} \frac{f(r \zeta)}{\operatorname{Re} f(0)}\right\}
\end{aligned}
$$


Consequently,

$$
\lim _{r \rightarrow 1}(1-r)^{k+1} f^{(k)}(r \zeta)=\bar{\zeta}^{k} 2 k ! \lim _{r \rightarrow 1} \frac{1-r}{1+r} f(r \zeta)
$$

The following is the proof of Theorem 1.1.

Proof. By considering the analytic function $\bar{\zeta} \varphi(\zeta z): \mathbb{D} \rightarrow \mathbb{D}$, we only need to prove Theorem 1.1 for the case $\zeta=1$ without loss of generality. Let

$$
f(z)=\frac{1+\varphi(z)}{1-\varphi(z)}, \quad z \in \mathbb{D} .
$$

Then

$$
\operatorname{Re} f(z)>0 \quad \text { and } \quad \varphi(z)=\frac{f(z)-1}{f(z)+1}, \quad \forall z \in \mathbb{D} .
$$

Furthermore,

$$
\lim _{r \rightarrow 1} \frac{1-r}{1+r} f(r)=\lim _{r \rightarrow 1} \frac{1-r}{1+r} \frac{1+\varphi(r)}{1-\varphi(r)}=\lim _{r \rightarrow 1} \frac{1-r}{1-\varphi(r)} \frac{1+\varphi(r)}{1+r}=\frac{1}{\varphi^{\prime}(1)} .
$$

Subsequently,

By Lemma 2.1,

$$
\lim _{r \rightarrow 1}(1-r)(f(r)+1)=\frac{2}{\varphi^{\prime}(1)}
$$

$$
\lim _{r \rightarrow 1}(1-r)^{k+1} \frac{f^{(k)}(r)}{k !}=\frac{1}{k !} \lim _{r \rightarrow 1} \frac{1-r}{1+r} f(r)=\frac{2}{\varphi^{\prime}(1)} \quad \text { for } \quad k \geq 0 .
$$

Let $h(z)=\frac{z-1}{z+1}$, then $\varphi(z)=h(f(z))$. By Faà di Bruno's formula [4],

$$
\varphi^{(\ell)}(r)=\frac{d^{\ell}}{d z^{\ell}} h(f(r))=\sum \frac{\ell !}{m_{1} ! m_{2} ! \cdots m_{\ell} !} h^{\left(m_{1}+\cdots+m_{\ell}\right)}(f(r)) \prod_{j}\left(\frac{f^{(j)}(r)}{j !}\right)^{m_{j}}
$$

where the sum is over all $\ell$-tuples $\left(m_{1}, m_{2}, \cdots, m_{\ell}\right)$ satisfying

$$
1 m_{1}+2 m_{2}+\cdots+\ell m_{\ell}=\ell .
$$

Since

we have

$$
h^{(k)}(z)=\frac{2(-1)^{k+1} k !}{(z+1)^{k+1}}, \quad k \geq 1
$$

$$
\begin{aligned}
\varphi^{(\ell)}(r) & =\sum \frac{\ell !}{m_{1} ! m_{2} ! \cdots m_{\ell} !} \frac{2(-1)^{\left(m_{1}+\cdots+m_{\ell}+1\right)}\left(m_{1}+\cdots+m_{\ell}\right) !}{(f(r)+1)^{m_{1}+\cdots+m_{\ell}+1}} \prod_{j}\left(\frac{f^{(j)}(r)}{j !}\right)^{m_{j}} \\
& =\ell ! \sum \frac{(-1)^{\left(m_{1}+\cdots+m_{\ell}+1\right)}\left(m_{1}+\cdots+m_{\ell}\right) !}{m_{1} ! m_{2} ! \cdots m_{\ell} !} \frac{2}{f(r)+1} \prod_{j}\left(\frac{f^{(j)}(r)}{(f(r)+1) j !}\right)^{m_{j}} .
\end{aligned}
$$


Notice that for each term of the sum, $1 m_{1}+2 m_{2}+\cdots+\ell m_{\ell}=\ell$, therefore

$$
\begin{aligned}
& \lim _{r \rightarrow 1}\left\{(1-r)^{\ell-1} \frac{2}{f(r)+1} \prod_{j}\left(\frac{f^{(j)}(r)}{(f(r)+1) j !}\right)^{m_{j}}\right\} \\
= & \lim _{r \rightarrow 1}\left\{\frac{2}{(1-r)(f(r)+1)} \prod_{j}\left(\frac{(1-r)^{j+1} f^{(j)}(r) / j !}{(1-r)(f(r)+1)}\right)^{m_{j}}\right\} \\
= & \frac{2}{2 / \varphi^{\prime}(1)} \prod_{j}\left(\frac{2 / \varphi^{\prime}(1)}{2 / \varphi^{\prime}(1)}\right)^{m_{j}}=\varphi^{\prime}(1) .
\end{aligned}
$$

Consequently,

$$
\lim _{r \rightarrow 1}\left\{(1-r)^{\ell-1} \varphi^{(\ell)}(r)\right\}=\varphi^{\prime}(1) \ell ! \sum \frac{(-1)^{\left(m_{1}+\cdots+m_{\ell}+1\right)}\left(m_{1}+\cdots+m_{\ell}\right) !}{m_{1} ! m_{2} ! \cdots m_{\ell} !} .
$$

To see that the above sum is zero, consider the function

$$
g(x)=x^{-1}, \quad g^{(k)}(x)=k !(-1)^{k} x^{-(k+1)}, \quad g^{(k)}(g(x))=-k !(-x)^{k+1}, \quad x \in(0,1] .
$$

Applying Faà di Bruno's formula to $x=g(g(x))$, we have

$$
\begin{aligned}
\frac{d^{\ell}}{d x^{\ell}}(x) & =\frac{d^{\ell}}{d r^{\ell}} g(g(x)) \\
& =\sum \frac{\ell !}{m_{1} ! m_{2} ! \cdots m_{\ell} !} g^{\left(m_{1}+\cdots+m_{\ell}\right)}(g(r)) \prod_{j}\left(\frac{g^{(j)}(r)}{j !}\right)^{m_{j}} \\
& =\ell ! \sum \frac{-\left(m_{1}+\cdots+m_{\ell}\right) !(-x)^{m_{1}+\cdots+m_{\ell}+1}}{m_{1} ! m_{2} ! \cdots m_{\ell} !} \prod_{j}\left(\frac{(-1)^{j}}{x^{j+1}}\right)^{m_{j}} \\
& =\ell ! \sum \frac{-\left(m_{1}+\cdots+m_{\ell}\right) !(-1)^{m_{1}+\cdots+m_{\ell}+1} x}{m_{1} ! m_{2} ! \cdots m_{\ell} !} \frac{(-1)^{\ell}}{x^{\ell}} \\
& =\left(\frac{-1}{x}\right)^{\ell-1} \ell ! \sum \frac{(-1)^{m_{1}+\cdots+m_{\ell}+1}\left(m_{1}+\cdots+m_{\ell}\right) !}{m_{1} ! m_{2} ! \cdots m_{\ell} !} \equiv 0, \quad \forall x \in(0,1], \ell \geq 2 .
\end{aligned}
$$

Hence,

$$
\begin{aligned}
\lim _{r \rightarrow 1}\left\{(1-r)^{\ell-1} \varphi^{(\ell)}(r)\right\} & =\varphi^{\prime}(1) \ell ! \sum \frac{(-1)^{\left(m_{1}+\cdots+m_{\ell}+1\right)}\left(m_{1}+\cdots+m_{\ell}\right) !}{m_{1} ! m_{2} ! \cdots m_{\ell} !} \\
& =\varphi^{\prime}(1)(-1)^{\ell-1}\left(\left.\frac{d^{\ell}}{d x^{\ell}}(x)\right|_{x=1}\right) \equiv 0
\end{aligned}
$$

therefore

$$
\varphi^{(\ell)}(r)=o\left(\frac{1}{(1-r)^{\ell-1}}\right) \quad \text { as } \quad r \rightarrow 1, \quad \forall \ell \geq 2
$$




\section{Proof of Proposition 1.2}

The following lemma is needed for the proof of Proposition 1.2.

Lemma 3.1. Let $\alpha \in(0,1)$,

$$
\begin{aligned}
f_{1}(x) & =\sum_{n=0}^{\infty} a_{2 n+1} x^{2 n+1}, \quad a_{2 n+1}=\left(\begin{array}{c}
\alpha \\
2 n+1
\end{array}\right)=\frac{\alpha(\alpha-1) \cdots(\alpha-2 n)}{(2 n+1) !}, \\
f_{2}(x) & =\sum_{n=0}^{\infty} b_{2 n} x^{2 n}, \quad b_{2 n}=\left(\begin{array}{c}
\alpha \\
2 n
\end{array}\right)=\frac{\alpha(\alpha-1) \cdots(\alpha-2 n+1)}{(2 n) !} \\
h(x) & =\frac{f_{1}(x)}{f_{2}(x)}=\sum_{n=0}^{\infty} c_{n} x^{n} .
\end{aligned}
$$

Then

$$
c_{2 n}=0, \quad c_{2 n+1}>0, \quad \forall n \geq 0 .
$$

Proof. By the symmetry of $f_{1}$ and $f_{2}$,

$$
h(-x)=\frac{f_{1}(-x)}{f_{2}(-x)}=-\frac{f_{1}(x)}{f_{2}(x)}=-h(x) \quad \Longrightarrow \quad c_{2 n}=0, \forall n \geq 0 .
$$

Furthermore,

$$
f_{1}(x)=h(x) f_{2}(x) \quad \Longrightarrow \quad a_{2 n+1}=c_{2 n+1} b_{0}+c_{2 n-1} b_{2}+\cdots+c_{1} b_{2 n}, \quad \forall n \geq 0 .
$$

Since $b_{0}=1$, we have

$$
c_{2 n+1}=a_{2 n+1}-\left(c_{2 n-1} b_{2}+\cdots+c_{1} b_{2 n}\right), \quad \forall n \geq 0 .
$$

For $n=0,1$,

$$
\begin{aligned}
& c_{1}=a_{1}=\alpha>0, \\
& c_{3}=a_{3}-c_{1} b_{2}=\frac{\alpha(\alpha-1)(\alpha-2)}{3 !}-\alpha \frac{\alpha(\alpha-1)}{2 !}=\alpha(\alpha-1)\left(-\frac{1}{3}\right)(\alpha+1)>0 .
\end{aligned}
$$

Now assume $c_{2 j-1}>0$ for $j=1, \cdots, k$ for some $k \geq 1$. Notice that for $\alpha \in(0,1)$,

$$
a_{2 k+1}>0, \forall k \geq 0 \quad \text { and } \quad b_{2 k}<0, \forall k \geq 1 .
$$

Therefore

$$
c_{2 k+1}=a_{2 k+1}-c_{2 k-1} b_{2}-\cdots-c_{1} b_{2 k}>0,
$$

because every term is positive. By induction, $c_{2 n+1}>0, \forall n \geq 0$.

The following is the proof of Proposition 1.2.

Proof. We prove (2) in Proposition 1.2 by constructing a function $\psi$ such that $\psi$ (or its rotation) satisfies (2). First consider

$$
\varphi(z)=\frac{1-z}{1+z}, \quad \operatorname{Re} \varphi(z)=\frac{1-|z|^{2}}{|1+z|^{2}}>0, \quad z \in \mathbb{D} .
$$


$\varphi$ is its own inverse:

$$
\varphi^{-1}=\varphi: \quad \varphi(\varphi(z))=\frac{1-\frac{1-z}{1+z}}{1+\frac{1-z}{1+z}}=z
$$

For $\alpha \in(0,1)$, let $g(z)=z^{\alpha}$, and define

$$
f=\varphi^{-1} \circ g \circ \varphi=\frac{1-\left(\frac{1-z}{1+z}\right)^{\alpha}}{1+\left(\frac{1-z}{1+z}\right)^{\alpha}}, \quad \mathbb{D} \rightarrow \mathbb{H} \rightarrow \mathbb{D} .
$$

Then

$$
f(0)=0, \quad f(1)=1, \quad f^{\prime}(z) \neq 0 .
$$

Therefore $f: \mathbb{D} \rightarrow \mathbb{D}$ is univalent and $z=1$ is a fixed point of $f$. Considering the Taylor expansions

$$
(1+z)^{\alpha}=\sum_{n=0}^{\infty}\left(\begin{array}{l}
\alpha \\
n
\end{array}\right) z^{n}, \quad(1-z)^{\alpha}=\sum_{n=0}^{\infty}(-1)^{n}\left(\begin{array}{l}
\alpha \\
n
\end{array}\right) z^{n}
$$

we have

$$
f(z)=\frac{(1+z)^{\alpha}-(1-z)^{\alpha}}{(1+z)^{\alpha}+(1-z)^{\alpha}}=\frac{\sum_{n=0}^{\infty}\left(\begin{array}{c}
\alpha \\
2 n+1
\end{array}\right) z^{2 n+1}}{\sum_{n=0}^{\infty}\left(\begin{array}{c}
\alpha \\
2 n
\end{array}\right) z^{2 n}}=\sum_{n=0}^{\infty} c_{n} z^{n}=\sum_{n=0}^{\infty} c_{2 n+1} z^{2 n+1} .
$$

Define

$$
F(z)=\int_{0}^{z} f(w) d w=\int_{0}^{z} \sum_{n=0}^{\infty} c_{2 n+1} w^{2 n+1} d w=\sum_{n=0}^{\infty} \frac{c_{2 n+1}}{2 n+2} z^{2 n+2}, \quad z \in \mathbb{D} .
$$

By Lemma 3.1, $c_{2 n+1}>0$ for $n \geq 0$. Therefore $|F(z)|$ achieves its maximum on the boundary at $z=1$ :

$|F(z)| \leq \sum_{n=0}^{\infty} \frac{c_{2 n+1}}{2 n+2}|z|^{2 n+2} \leq \sum_{n=0}^{\infty} \frac{c_{2 n+1}}{2 n+1}|z|^{2 n+1} \leq \sum_{n=0}^{\infty} \frac{c_{2 n+1}}{2 n+1}=F(1)=\max _{|z| \leq 1}|F(z)|$.

By the maximal principle, the function

$$
\psi(z)=\frac{F(z)}{F(1)}, \quad z \in \mathbb{D}
$$

maps $\mathbb{D}$ into $\mathbb{D}$. Furthermore, $z=1$ is a fixed point of $\psi$,

$$
\psi(1)=1, \quad \psi^{\prime}(1)=\frac{F^{\prime}(1)}{F(1)}=\frac{f(1)}{F(1)} \neq \infty, \quad \psi^{\prime \prime}(1)=\frac{f^{\prime}(1)}{F(1)}=\infty
$$

and

$$
\psi^{(k)}(r)=\frac{f^{(k-1)}(r)}{F(1)}, \quad k \geq 2
$$


Notice that

$$
\frac{d}{d z}\left(\frac{1-z}{1+z}\right)^{\alpha}=\alpha\left(\frac{1-z}{1+z}\right)^{\alpha-1} \frac{-2}{(1+z)^{2}}=\alpha\left(\frac{1-z}{1+z}\right)^{\alpha-1} \varphi^{\prime}(z),
$$

and

$$
\frac{d^{2}}{d z^{2}}\left(\frac{1-z}{1+z}\right)^{\alpha}=\alpha(\alpha-1)\left(\frac{1-z}{1+z}\right)^{\alpha-2}\left(\varphi^{\prime}(z)\right)^{2}+\alpha\left(\frac{1-z}{1+z}\right)^{\alpha-1} \varphi^{\prime \prime}(z),
$$

etc. Using the big $O$ notation for $z$ near 1 , we may write

$$
\frac{d^{k}}{d z^{k}}\left(\frac{1-z}{1+z}\right)^{\alpha}=k !\left(\begin{array}{l}
\alpha \\
k
\end{array}\right)\left(\frac{1-z}{1+z}\right)^{\alpha-k}\left(\varphi^{\prime}(z)\right)^{k}+O\left((1-z)^{\alpha-k+1}\right) .
$$

Applying the little $o$ notation, we have

$$
\begin{aligned}
\frac{d^{k}}{d z^{k}} g(\varphi(r)) & =\left.\frac{d^{k}}{d z^{k}}\left(\frac{1-z}{1+z}\right)^{\alpha}\right|_{z=r} \\
& =k !\left(\begin{array}{l}
\alpha \\
k
\end{array}\right)\left(\frac{1-r}{1+r}\right)^{\alpha-k}\left(\frac{-2}{(1+r)^{2}}\right)^{k}+O\left((1-r)^{\alpha-k+1}\right) \\
& =k !\left(\begin{array}{l}
\alpha \\
k
\end{array}\right)(1-r)^{\alpha-k}(-1)^{k} \frac{2^{k}}{(1+r)^{\alpha+k}}+o\left((1-r)^{\alpha-k}\right)
\end{aligned}
$$

By Faà di Bruno's formula [4],

$$
f^{(\ell)}(r)=\frac{d^{\ell}}{d z^{\ell}} \varphi(g(\varphi(r)))=\sum \frac{\ell !}{m_{1} ! m_{2} ! \cdots m_{\ell} !} \varphi^{\left(m_{1}+\cdots+m_{\ell}\right)}\left(g(\varphi(r)) \prod_{j}\left(\frac{\frac{d^{k}}{d z^{k}} g(\varphi(r))}{j !}\right)^{m_{j}}\right.
$$

where the sum is over all $\ell$-tuples $\left(m_{1}, m_{2}, \cdots, m_{\ell}\right)$ satisfying

$$
1 m_{1}+2 m_{2}+\cdots+\ell m_{\ell}=\ell .
$$

Notice that

$$
\varphi^{(k)}(z)=2(-1)^{k} k !(1+z)^{-(k+1)}, \quad \varphi^{(k)}\left(g(\varphi(r))=2(-1)^{k} k !\left(1+\left(\frac{1-r}{1+r}\right)^{\alpha}\right)^{-(k+1)},\right.
$$

and

$$
\begin{aligned}
\prod_{j}\left(\frac{\frac{d^{k}}{d z^{k}} g(\varphi(r))}{j !}\right)^{m_{j}} & =\prod_{j}\left\{\left(\begin{array}{c}
\alpha \\
j
\end{array}\right)(1-r)^{\alpha-j}(-1)^{j} \frac{2^{j}}{(1+r)^{\alpha+j}}+o\left((1-r)^{\alpha-j}\right)\right\}^{m_{j}} \\
= & (-1)^{m_{1}+\cdots+m_{\ell}}(1-r)^{\alpha-\ell}\left(\frac{2}{1+r}\right)^{\ell} \prod_{j}\left\{\left(\begin{array}{c}
\alpha \\
j
\end{array}\right) \frac{(-1)^{j-1}}{(1+r)^{\alpha}}\right\} \\
& +o\left((1-r)^{\alpha-\ell}\right),
\end{aligned}
$$


where each term in the product

$$
\left(\begin{array}{c}
\alpha \\
j
\end{array}\right) \frac{(-1)^{j-1}}{(1+r)^{\alpha}}=\frac{\alpha(\alpha-1) \cdots(\alpha-j+1)(-1)^{j-1}}{j !(1+r)^{\alpha}}=\frac{\alpha(1-\alpha) \cdots(j-1-\alpha)}{j !(1+r)^{\alpha}}>0
$$

for $\alpha \in(0,1)$, and the little $o$ term is obtained by the fact that

$$
\lim _{r \rightarrow 1} \frac{\prod_{j}(1-r)^{\alpha-j}}{(1-r)^{\alpha-\ell}}=\lim _{r \rightarrow 1} \frac{(1-r)^{\left(m_{1}+\cdots+m_{\ell}\right) \alpha-\ell}}{(1-r)^{\alpha-\ell}}=\lim _{r \rightarrow 1}(1-r)^{\left(m_{1}+\cdots+m_{\ell}-1\right) \alpha}=0 .
$$

Since for given $\alpha \in(0,1)$ and $\ell \geq 1$,

$$
\sum \frac{\ell ! 2\left(m_{1}+\cdots+m_{\ell}\right) !}{m_{1} ! m_{2} ! \cdots m_{\ell} !}\left(1+\left(\frac{1-r}{1+r}\right)^{\alpha}\right)^{-\left(m_{1}+\cdots+m_{\ell}+1\right)}
$$

is bounded and $>0$ as $r \rightarrow 1$, we have

$$
\begin{aligned}
& f^{(\ell)}(r)=\sum \frac{\ell ! 2\left(m_{1}+\cdots+m_{\ell}\right) !}{m_{1} ! m_{2} ! \cdots m_{\ell} !}\left(1+\left(\frac{1-r}{1+r}\right)^{\alpha}\right)^{-\left(m_{1}+\cdots+m_{\ell}+1\right)}(1-r)^{\alpha-\ell}\left(\frac{2}{1+r}\right)^{\ell} \prod_{j}\left(\begin{array}{l}
\alpha \\
j
\end{array}\right) \frac{(-1)^{j-1}}{(1+r)^{\alpha}} \\
& +o\left((1-r)^{\alpha-\ell}\right) \text {. }
\end{aligned}
$$

Consequently

$\lim _{r \rightarrow 1}(1-r)^{\ell-\alpha} f^{(\ell)}(r)=\sum \frac{\ell ! 2\left(m_{1}+\cdots+m_{\ell}\right) !}{m_{1} ! m_{2} ! \cdots m_{\ell} !} \prod_{j}\left(\begin{array}{c}\alpha \\ j\end{array}\right) \frac{(-1)^{j-1}}{2^{\alpha}}=C_{\ell, \alpha}>0$,

where $C_{\ell, \alpha}$ is a constant for any given $\alpha \in(0,1)$ and $\ell \geq 1$. Therefore we have

$$
\lim _{r \rightarrow 1}(1-r)^{n-1-\alpha} \psi^{(n)}(r)=\lim _{r \rightarrow 1}(1-r)^{n-1-\alpha} \frac{f^{(n-1)}(r)}{F(1)}=\frac{C_{n-1, \alpha}}{F(1)}>0, \quad \forall n \geq 2 .
$$

We have shown that (2) in Proposition 1.2 holds for $\psi$ with $\zeta=1$. For an arbitrary $\zeta \in \mathbb{T},(2)$ is satisfied by $\bar{\zeta} \psi(\zeta z)$.

To prove (3), we show that for any $m \geq 1$, there exists a function $\psi_{m}$ such that $\psi_{m}$ (or its rotation) satisfies the conditions in (3). Let

$$
\psi_{m}(z)=\frac{F_{m}(z)}{F_{m}(1)}, \quad F_{j}(z)=\int_{0}^{z} \frac{F_{j-1}(w)}{F_{j-1}(1)} d w, \quad 1 \leq j \leq m
$$

where

$$
F_{1}=F, \quad F_{0}=f, \quad \psi_{1}=\psi
$$


are the functions used in the above proof of (2). By the construction,

$$
\begin{aligned}
F_{1}(z) & =\sum_{n=0}^{\infty} \frac{c_{2 n+1}}{2 n+2} z^{2 n+2} \\
\left|F_{1}(z)\right| & =\sum_{n=0}^{\infty} \frac{c_{2 n+1}}{2 n+2}|z|^{2 n+2} \leq \sum_{n=0}^{\infty} \frac{c_{2 n+1}}{2 n+2}=F_{1}(1) \\
F_{2}(z) & =\int_{0}^{z} \frac{F_{1}(w)}{F_{1}(1)} d w=\frac{1}{F_{1}(1)} \sum_{n=0}^{\infty} \frac{c_{2 n+1}}{(2 n+2)(2 n+3)} z^{2 n+3}, \\
\left|F_{2}(z)\right| & =\frac{1}{F_{1}(1)} \sum_{n=0}^{\infty} \frac{c_{2 n+1}}{(2 n+2)(2 n+3)}|z|^{2 n+3} \\
& \leq \frac{1}{F_{1}(1)} \sum_{n=0}^{\infty} \frac{c_{2 n+1}}{(2 n+2)(2 n+3)}=F_{2}(1),
\end{aligned}
$$

etc. For $j=1,2, \cdots, m$,

$$
\begin{aligned}
F_{j}(z) & =\int_{0}^{z} \frac{F_{j-1}(w)}{F_{j-1}(1)} d w=\frac{1}{F_{j-1}(1)} \sum_{n=0}^{\infty} \frac{c_{2 n+1}}{(2 n+2)(2 n+3) \cdots(2 n+1+j)} z^{2 n+1+j} \\
\left|F_{j}(z)\right| & =\frac{1}{F_{j-1}(1)} \sum_{n=0}^{\infty} \frac{c_{2 n+1}}{(2 n+2)(2 n+3) \cdots(2 n+1+j)}|z|^{2 n+1+j} \\
& \leq \frac{1}{F_{j-1}(1)} \sum_{n=0}^{\infty} \frac{c_{2 n+1}}{(2 n+2)(2 n+3) \cdots(2 n+1+j)}=F_{j}(1) .
\end{aligned}
$$

By the maximal principle, the functions

$$
\psi_{m}(z)=\frac{F_{m}(z)}{F_{m}(1)}, \quad \psi_{m}(1)=1
$$

map $\mathbb{D}$ into $\mathbb{D}$. Furthermore,

$$
\begin{aligned}
\psi_{m}^{(k)}(1) & =\frac{F_{m}^{(k)}(1)}{F_{m}(1)}=\frac{F_{m-1}^{(k-1)}(1)}{F_{m}(1) F_{m-1}(1)}=\frac{F_{m-2}^{(k-2)}(1)}{F_{m}(1) F_{m-1}(1) F_{m-2}(1)} \\
& =\frac{F_{m-k}(1)}{\prod_{j=0}^{k} F_{m-j}(1)}<\infty, \quad k=1,2, \cdots, m,
\end{aligned}
$$

especially,

$$
\psi_{m}^{(m)}(1)=\frac{f(1)}{\prod_{j=1}^{m} F_{j}(1)}<\infty .
$$

Notice that

$$
\psi_{m}^{(m)}(z)=\frac{\psi(z)}{\prod_{j=1}^{m} F_{j}(1)}<\infty .
$$


Consequently the proven result (2) implies (3) for $\zeta=1$. For an arbitrary $\zeta \in \mathbb{T}$, (3) is satisfied by $\bar{\zeta} \psi_{m}(\zeta z)$.

This completes the proof of Proposition 1.2.

\section{Proof of Theorem 1.3}

We need several lemmas to prove Theorem 1.3.

Lemma 4.1. If $\varphi(x)=x^{2}+a x+b$, then for any $m \geq 0$,

$$
\frac{d^{\ell}}{d x^{\ell}} h(\varphi(x))= \begin{cases}\sum_{j=0}^{m} \frac{(2 m) !}{(2 j) !(m-j) !} h^{(m+j)}(\varphi)\left(\varphi^{\prime}\right)^{2 j}, & \ell=2 m ; \\ \sum_{j=0}^{m} \frac{(2 m+1) !}{(2 j+1) !(m-j) !} h^{(m+j+1)}(\varphi)\left(\varphi^{\prime}\right)^{2 j+1}, & \ell=2 m+1 .\end{cases}
$$

Proof. Again by Faà di Bruno's formula [4],

$$
\frac{d^{\ell}}{d x^{\ell}} h(\varphi(x))=\sum \frac{\ell !}{m_{1} ! m_{2} ! \cdots m_{\ell} !} h^{\left(m_{1}+\cdots+m_{\ell}\right)}(\varphi(x)) \prod_{j}\left(\frac{\varphi^{(j)}(x)}{j !}\right)^{m_{j}}
$$

where the sum is over all $\ell$-tuples $\left(m_{1}, m_{2}, \cdots, m_{\ell}\right)$ satisfying

$$
1 m_{1}+2 m_{2}+\cdots+\ell m_{\ell}=\ell .
$$

Since $\varphi^{\prime \prime} \equiv 2, \varphi^{(j)}=0$ for $j \geq 3$, the product in Faà di Bruno's formula simplifies to

$$
\prod_{j}\left(\frac{\varphi^{(j)}(x)}{j !}\right)^{m_{j}}=\varphi^{\prime}(x)^{m_{1}} \quad\left(\text { with } 0^{0}=1\right)
$$

which implies

$$
\frac{d^{\ell}}{d x^{\ell}} h(\varphi(x))=\sum_{m_{1}+2 m_{2}=\ell} \frac{\ell !}{m_{1} ! m_{2} !} h^{\left(m_{1}+m_{2}\right)}(\varphi)\left(\varphi^{\prime}\right)^{m_{1}},
$$

where

$$
m_{2}= \begin{cases}m-\frac{m_{1}}{2}, & \ell=2 m \\ m-\frac{m_{1}-1}{2}, & \ell=2 m+1\end{cases}
$$

Relabeling the summation index by $j$,

$$
j=\left\{\begin{array}{ll}
\frac{m_{1}}{2}, \quad \ell=2 m ; \\
\frac{m_{1}-1}{2}, \quad \ell=2 m+1,
\end{array} \quad \text { then } \quad m_{1}+m_{2}= \begin{cases}m+j, & \ell=2 m ; \\
m+j+1, & \ell=2 m+1 .\end{cases}\right.
$$

Replacing $m_{1}$ and $m_{2}$ by $j$ and $m,(7)$ becomes (6). 
Lemma 4.2. Let $f(r)=\frac{1}{|r \zeta-\eta|^{n}}, r \in[0,1), \zeta, \eta \in S^{n-1}, n \geq 2$. Let $\theta_{r} \in[0, \pi]$ denote the angle between the n-vectors $\zeta$ and $r \zeta-\eta$ for any $r \in[0,1]$. Then

$f^{(\ell)}(r)= \begin{cases}\frac{1}{|r \zeta-\eta|^{n+\ell}} \sum_{j=0}^{m} \frac{\left(\cos \theta_{r}\right)^{2 j}(2 m) !}{(2 j) !(m-j) !} \frac{(-1)^{m+j} n(n+2) \cdots(n+2 m+2 j-2)}{2^{m-j}}, & \ell=2 m ; \\ \frac{r-\zeta \cdot \eta}{|r \zeta-\eta|^{n+\ell+1}} \sum_{j=0}^{m} \frac{\left(\cos \theta_{r}\right)^{2 j}(2 m+1) !}{(2 j+1) !(m-j) !} \frac{(-1)^{m+j+1} n(n+2) \cdots(n+2 m+2 j)}{2^{m-j}}, & \ell=2 m+1 .\end{cases}$

Proof. Let $h(x)=x^{-n / 2}, \varphi(r)=|r \zeta-\eta|^{2}$. Then

$$
\begin{aligned}
\varphi^{\prime}(r)=\frac{d}{d r} \sum_{j=1}^{n}\left(r \zeta_{j}-\right. & \left.\eta_{j}\right)^{2}=2(r-\zeta \cdot \eta), \quad \varphi^{\prime \prime}(r)=2, \quad \varphi^{(j)}(r)=0 \text { for } j \geq 3, \\
h^{(\alpha)}(x) & =\left(-\frac{n}{2}\right)\left(-\frac{n}{2}-1\right) \cdots\left(-\frac{n}{2}-(\alpha-1)\right) x^{-\frac{n}{2}-\alpha} \\
& =(-1)^{\alpha} \frac{n(n+2) \cdots(n+2(\alpha-1))}{2^{\alpha}} \frac{1}{x^{(n+2 \alpha) / 2}} .
\end{aligned}
$$

Denote $\zeta=\left(\zeta_{1}, \cdots, \zeta_{n}\right), \eta=\left(\eta_{1}, \cdots, \eta_{n}\right)$ and $\zeta \cdot \eta=\sum_{j} \zeta_{j} \eta_{j}$ the Euclidean inner product of $\zeta$ and $\eta$. Notice that

$$
(r-\zeta \cdot \eta)^{2}=|r \zeta \cdot \zeta-\zeta \cdot \eta|^{2}=|\zeta \cdot(r \zeta-\eta)|^{2}=|r \zeta-\eta|^{2} \cos ^{2} \theta_{r}
$$

therefore

$$
\begin{aligned}
h^{(m+j)}(\varphi(r))\left(\varphi^{\prime}(r)\right)^{2 j} & =(-1)^{m+j} \frac{n(n+2) \cdots(n+2(m+j-1))}{2^{m+j}} \frac{2^{2 j}(r-\zeta \cdot \eta)^{2 j}}{|r \zeta-\eta|^{n+2 m+2 j}} \\
& =\frac{\left(\cos \theta_{r}\right)^{2 j}}{|r \zeta-\eta|^{n+2 m}}(-1)^{m+j} \frac{n(n+2) \cdots(n+2 m+2 j-2)}{2^{m-j}},
\end{aligned}
$$

and

$$
\begin{aligned}
h^{(m+j+1)}(\varphi(r))\left(\varphi^{\prime}(r)\right)^{2 j+1} & =(-1)^{m+j+1} \frac{n(n+2) \cdots(n+2(m+j))}{2^{m+j+1}} \frac{2^{2 j+1}(r-\zeta \cdot \eta)^{2 j+1}}{|r \zeta-\eta|^{n+2 m+2 j+2}} \\
& =\frac{(r \zeta-\eta)\left(\cos \theta_{r}\right)^{2 j}}{|r \zeta-\eta|^{n+2 m+2}}(-1)^{m+j+1} \frac{n(n+2) \cdots(n+2 m+2 j)}{2^{m-j}} .
\end{aligned}
$$

Applying Lemma 4.1 to $f(r)=h(\varphi(r))$, the result of Lemma 4.2 follows.

Notation. Denote

$$
C_{n, k}=(-1)^{k} 2(C(n, k)+k C(n, k-1)) \quad \text { for } \quad k \geq 1, n \geq 2,
$$

where

(8) $C(n, \ell)= \begin{cases}\sum_{j=0}^{m} \frac{(2 m) !}{(2 j) !(m-j) !} \frac{(-1)^{m+j} n(n+2) \cdots(n+2 m+2 j-2)}{2^{m-j}}, & \ell=2 m ; \\ \sum_{j=0}^{m} \frac{(2 m+1) !}{(2 j+1) !(m-j) !} \frac{(-1)^{m+j+1} n(n+2) \cdots(n+2 m+2 j)}{2^{m-j}}, & \ell=2 m+1 ;\end{cases}$ 
for $m \geq 0$, with $C(n, 0)=1$.

Lemma 4.3. Let $\zeta, \eta \in S^{n-1}, n \geq 2$. Then for $k \geq 1$,

$$
\lim _{r \rightarrow 1}\left\{(1-r)^{n+k-1} \frac{d^{k}}{d r^{k}}\left(\frac{1-r^{2}}{|r \zeta-\eta|^{n}}\right)\right\}= \begin{cases}0, & \zeta \neq \eta \\ C_{n, k}, & \zeta=\eta\end{cases}
$$

Proof. Let $g(r)=1-r^{2}, f(r)=|r \zeta-\eta|^{-n}$. Then

$$
g^{\prime}(r)=-2 r, \quad g^{\prime \prime}(r)=-2 \quad \text { and } \quad g^{(j)}(r) \equiv 0, j \geq 3 .
$$

For $k \geq 1$,

$$
\begin{aligned}
\frac{d^{k}}{d r^{k}}\left(\frac{1-r^{2}}{|r \zeta-\eta|^{n}}\right) & =\frac{d^{k}}{d r^{k}}(f(r) g(r))=\sum_{j=0}^{k}\left(\begin{array}{c}
k \\
j
\end{array}\right) f^{(k-j)}(r) g^{(j)}(r) \\
& =\left(\begin{array}{c}
k \\
0
\end{array}\right) f^{(k)}(r) g(r)+\left(\begin{array}{c}
k \\
1
\end{array}\right) f^{(k-1)}(r) g^{\prime}(r)+\left(\begin{array}{c}
k \\
2
\end{array}\right) f^{(k-2)}(r) g^{\prime \prime}(r) \\
& =\left(1-r^{2}\right) f^{(k)}(r)-2 r k f^{(k-1)}(r)-k(k-1) f^{(k-2)}(r)
\end{aligned}
$$

with the convention that $f^{(\alpha)}(r)=g^{(\alpha)}(r) \equiv 0$ for $\alpha<0$. Let

$$
C(n, \ell, \theta)= \begin{cases}\sum_{j=0}^{m} \frac{(\cos \theta)^{2 j}(2 m) !}{(2 j) !(m-j) !} \frac{(-1)^{m+j} n(n+2) \cdots(n+2 m+2 j-2)}{2^{m-j}}, & \ell=2 m ; \\ \sum_{j=0}^{m} \frac{(\cos \theta)^{2 j}(2 m+1) !}{(2 j+1) !(m-j) !} \frac{(-1)^{m+j+1} n(n+2) \cdots(n+2 m+2 j)}{2^{m-j}}, & \ell=2 m+1 ;\end{cases}
$$

Then

From Lemma 4.2,

$$
C(n, \ell)=C(n, \ell, 0), \quad \ell \geq 1, n \geq 2
$$

$f^{(\ell)}(r)=\frac{A(\ell, r, \zeta, \eta)}{|r \zeta-\eta|^{n+\ell}} C\left(n, \ell, \theta_{r}\right), \quad$ where $\quad A(\ell, r, \zeta, \eta)= \begin{cases}1, & \ell \text { even; } \\ \frac{r-\zeta \cdot \eta}{|r \zeta-\eta|}, & \ell \text { odd } .\end{cases}$

Thus

$$
\begin{aligned}
& \lim _{r \rightarrow 1}\left\{(1-r)^{n+k-1} \frac{d^{k}}{d r^{k}}\left(\frac{1-r^{2}}{|r \zeta-\eta|^{n}}\right)\right\} \\
= & \lim _{r \rightarrow 1}\left\{(1-r)^{n+k-1}\left[\left(1-r^{2}\right) f^{(k)}(r)-2 r k f^{(k-1)}(r)-k(k-1) f^{(k-2)}(r)\right]\right\} \\
= & \lim _{r \rightarrow 1}\left\{\frac{\left(1-r^{2}\right)(1-r)^{n+k-1} A(k, r, \zeta, \eta)}{|r \zeta-\eta|^{n+k}} C\left(n, k, \theta_{r}\right)\right. \\
& -\frac{2 r k(1-r)^{n+k-1} A(k-1, r, \zeta, \eta)}{|r \zeta-\eta|^{n+k-1}} C\left(n, k-1, \theta_{r}\right) \\
& \left.-\frac{k(k-1)(1-r)^{n+k-1} A(k-2, r, \zeta, \eta)}{|r \zeta-\eta|^{n+k-2}} C\left(n, k-2, \theta_{r}\right)\right\} .
\end{aligned}
$$


Notice that $|A(k, r, \zeta, \eta)| \leq 1$ is bounded, $\theta_{r} \rightarrow 0$ as $r \rightarrow 1$, and $C(n, \ell, 0)$ is bounded, hence the last term $\rightarrow 0$ as $r \rightarrow 1$. In addition,

$\lim _{r \rightarrow 1} \frac{1-r}{|r \zeta-\eta|}=\left\{\begin{array}{ll}0, & \zeta \neq \eta ; \\ 1, & \zeta=\eta ;\end{array} \quad\right.$ and $\quad \lim _{r \rightarrow 1} \frac{(1-r) A(k, r, \zeta, \eta)}{|r \zeta-\eta|}= \begin{cases}0, & \zeta \neq \eta \\ (-1)^{k}, & \zeta=\eta\end{cases}$

so we have

$$
\begin{aligned}
& \lim _{r \rightarrow 1}\left\{(1-r)^{n+k-1} \frac{d^{k}}{d r^{k}}\left(\frac{1-r^{2}}{|r \zeta-\eta|^{n}}\right)\right\} \\
= & \lim _{r \rightarrow 1}\left\{\frac{(1+r)(1-r)^{n+k} A(k, r, \zeta, \eta)}{|r \zeta-\eta|^{n+k}} C\left(n, k, \theta_{r}\right)\right. \\
& \left.-\frac{2 r k(1-r)^{n+k-1} A(k-1, r, \zeta, \eta)}{|r \zeta-\eta|^{n+k-1}} C\left(n, k-1, \theta_{r}\right)\right\} \\
= & \begin{cases}0, & \zeta \neq \eta ; \\
2(-1)^{k} C(n, k, 0)-2(-1)^{k-1} k C(n, k-1,0), & \zeta=\eta ;\end{cases} \\
= & \begin{cases}0, & \zeta \neq \eta ; \\
2(-1)^{k} C(n, k)-2(-1)^{k-1} k C(n, k-1)=C_{n, k}, & \zeta=\eta .\end{cases}
\end{aligned}
$$

Lemma 4.4. $\quad C_{n, k}=2 \frac{(n+k-2) !}{(n-2) !}$ for $k \geq 1, n \geq 2$.

We postpone the proof of Lemma 4.4 until after the proof of Theorem 1.3.

Now we prove Theorem 1.3.

Proof. For any $x \in B^{n}, x=r \zeta, \zeta \in S^{n-1}, n \geq 2$, we may write

$$
u(r \zeta)=\int_{S^{n-1}} \frac{1-r^{2}}{|r \zeta-\eta|^{n}} d \mu(\eta)
$$

By Lemma 4.3 , for any $k \geq 1$,

$$
\int_{S^{n-1}} \lim _{r \rightarrow 1}\left\{(1-r)^{n+k-1} \frac{d^{k}}{d r^{k}}\left(\frac{1-r^{2}}{|r \zeta-\eta|^{n}}\right)\right\} d \mu(\eta)= \begin{cases}0, & \mu(\{\zeta\})=0 \\ C_{n, k} \mu(\{\zeta\}), & \mu(\{\zeta\})>0\end{cases}
$$

By the interchangeability of differentiation and integration when the integral of the derivative converges and the Lebesgue's dominated convergence theorem, we 
have

$$
\begin{aligned}
\lim _{r \rightarrow 1}\left\{(1-r)^{n+k-1} \frac{d^{k}}{d r^{k}} u(r \zeta)\right\} & =\lim _{r \rightarrow 1}\left\{(1-r)^{n+k-1} \frac{d^{k}}{d r^{k}} \int_{S^{n-1}} \frac{1-r^{2}}{|r \zeta-\eta|^{n}} d \mu(\eta)\right\} \\
& =\lim _{r \rightarrow 1}\left\{(1-r)^{n+k-1} \int_{S^{n-1}} \frac{d^{k}}{d r^{k}}\left(\frac{1-r^{2}}{|r \zeta-\eta|^{n}}\right) d \mu(\eta)\right\} \\
& =\int_{S^{n-1}} \lim _{r \rightarrow 1}\left\{(1-r)^{n+k-1} \frac{d^{k}}{d r^{k}}\left(\frac{1-r^{2}}{|r \zeta-\eta|^{n}}\right)\right\} d \mu(\eta) \\
& =C_{n, k} \mu(\{\zeta\}) .
\end{aligned}
$$

By Theorem 1.1 in [5] (or by going through the proof of Lemma 4.3 with $k=0$ ),

$$
\lim _{r \rightarrow 1}\left\{(1-r)^{n-1} u(r \zeta)\right\}=2 \mu(\{\zeta\}) .
$$

Thus

$$
\lim _{r \rightarrow 1}\left\{\frac{(1-r)^{n-1}}{1+r} u(r \zeta)\right\}=\mu(\{\zeta\})
$$

and

$$
\lim _{r \rightarrow 1}\left\{(1-r)^{n+k-1} \frac{d^{k}}{d r^{k}} u(r \zeta)\right\}=C_{n, k} \lim _{r \rightarrow 1}\left\{\frac{(1-r)^{n-1}}{1+r} u(r \zeta)\right\} .
$$

So (4) holds by Lemma 4.4. Consequently (5) holds because each positive harmonic function in the unit ball corresponds to a positive measure on the sphere (ref. [1]), and that the set of non-zero point mass of the measure is countable.

This completes the proof of Theorem 1.3.

The following is the proof of Lemma 4.4.

Proof. Lemma 4.4 is proved by induction. By the definition,

$$
\begin{aligned}
& C(n, 0)=1, \\
& C(n, 1)=\frac{(-1)^{1} n}{2^{0}}=-n, \\
& C(n, 2)=\frac{2 !(-1)^{1} n}{2^{1}}+\frac{2 !(-1)^{2} n(n+2)}{2 ! 2^{0}}=-n+n(n+2)=n(n+1) .
\end{aligned}
$$

For $k=1$ and 2 ,

$$
\begin{aligned}
& C_{n, 1}=(-1)^{1} 2(C(n, 1)+C(n, 0))=-2(-n+1)=2 \frac{(n+1-2) !}{(n-2) !}, \\
& C_{n, 2}=(-1)^{2} 2(C(n, 2)+2 C(n, 1))=2(n(n+1)-2 n)=2 \frac{(n+2-2) !}{(n-2) !} .
\end{aligned}
$$

For any $k$, assuming

$$
C_{n, k}=(-1)^{k} 2(C(n, k)+k C(n, k-1))=2 \frac{(n+k-2) !}{(n-2) !}
$$


we will prove

$$
C_{n, k+1}=(-1)^{k+1} 2(C(n, k+1)+(k+1) C(n, k))=2 \frac{(n+k-1) !}{(n-2) !} .
$$

Using the induction assumption, the above equation can be written as

$$
\begin{aligned}
(-1)^{k+1}(C(n, k+1)+(k+1) C(n, k)) & =\frac{(n+k-1)(n+k-2) !}{(n-2) !} \\
& =(n+k-1) C_{n, k} \\
& =(n+k-1)(-1)^{k}(C(n, k)+k C(n, k-1)),
\end{aligned}
$$

so it is sufficient to show

$$
-(C(n, k+1)+(k+1) C(n, k))=(n+k-1)(C(n, k)+k C(n, k-1))
$$

Write

$$
C(n, k)=\sum_{j} C(n, k, j)
$$

Notice that

$$
\begin{aligned}
C(n, 2 m+2, j) & =\frac{(m+1)(2 j+1)}{m+1-j} C(n, 2 m+1, j), \quad 0 \leq j \leq m ; \\
C(n, 2 m+2, j+1) & =-\frac{(m+1)(n+2 m+2 j+2)}{j+1} C(n, 2 m+1, j), \quad 0 \leq j \leq m .
\end{aligned}
$$

Therefore,

$$
\begin{aligned}
C(n, 2 m+2) & =\sum_{j=0}^{m+1}\left(\frac{m+1-j}{m+1}+\frac{j}{m+1}\right) C(n, 2 m+2, j) \\
& =\sum_{j=0}^{m} \frac{m+1-j}{m+1} C(n, 2 m+2, j)+\sum_{j=1}^{m+1} \frac{j}{m+1} C(n, 2 m+2, j) \\
& =\sum_{j=0}^{m} \frac{m+1-j}{m+1} C(n, 2 m+2, j)+\sum_{j=0}^{m} \frac{j+1}{m+1} C(n, 2 m+2, j+1) \\
& =\sum_{j=0}^{m}(2 j+1) C(n, 2 m+1, j)-\sum_{j=0}^{m}(n+2 m+2 j+2) C(n, 2 m+1, j) \\
& =-\sum_{j=0}^{m}(n+2 m+1) C(n, 2 m+1, j) \\
& =-(n+2 m+1) C(n, 2 m+1) \quad \text { for } \quad m \geq 0 .
\end{aligned}
$$


Similarly,

$$
\begin{aligned}
& C(n, 2 m+1, j)=-\frac{(2 m+1)(n+2 m+2 j)}{2 j+1} C(n, 2 m, j), \quad 0 \leq j \leq m ; \\
& C(n, 2 m+1, j)=\frac{(2 m+1)(2 j+2)}{2 m-2 j} C(n, 2 m, j+1), \quad 0 \leq j \leq m-1 .
\end{aligned}
$$

Consequently,

$$
\begin{aligned}
C(n, 2 m+1) & =\sum_{j=0}^{m} \frac{2 j+1}{2 m+1} C(n, 2 m+1, j)+\sum_{j=0}^{m-1} \frac{2 m-2 j}{2 m+1} C(n, 2 m+1, j) \\
& =-\sum_{j=0}^{m}(n+2 m+2 j) C(n, 2 m, j)+\sum_{j=0}^{m-1}(2 j+2) C(n, 2 m, j+1) \\
& =-\sum_{j=0}^{m}(n+2 m+2 j) C(n, 2 m, j)+\sum_{j=0}^{m}(2 j) C(n, 2 m, j) \\
& =-\sum_{j=0}^{m}(n+2 m) C(n, 2 m, j) \\
& =-(n+2 m) C(n, 2 m) \quad \text { for } \quad m \geq 0 .
\end{aligned}
$$

The above relations between the adjacent $C(n, \ell)$ 's can be summarized as

$$
C(n, \ell)=-(n+\ell-1) C(n, \ell-1) \quad \text { for } \quad \ell \geq 1 .
$$

Applying the above equation repeatedly, for any $k \geq 1$ we have

$$
\begin{aligned}
\text { L.H.S. of }(9) & =-C(n, k+1)-(k+1) C(n, k) \\
& =(n+k) C(n, k)-(k+1) C(n, k)=(n-1) C(n, k),
\end{aligned}
$$

and

$$
\begin{aligned}
\text { R.H.S. of }(9) & =(n+k-1)(C(n, k)+k C(n, k-1)) \\
& =(n+k-1) C(n, k)+k(n+k-1) C(n, k-1) \\
& =(n+k-1) C(n, k)-k C(n, k)=(n-1) C(n, k) .
\end{aligned}
$$

Therefore (9) holds for all $k \geq 1$. This completes the proof of Lemma 4.4.

Acknowledgments. We thank Pietro Poggi-Corradini for his valuable idea for Proposition 1.2.

\section{REFERENCES}

[1] S. Axler, P. Bourdon and W. Ramey, Harmonic Function Theory, 2nd ed. Springer, New York, 2001.

[2] S. DAI and Y. PAN. Note on Schwarz-Pick estimates for bounded and positive real part analytic functions. Proc. Amer. Math. Soc. To appear. 
[3] P. Duren, Univalent functions, Springer, New York (2001).

[4] W. P. Johnson. The curious history of Faà di Bruno's formula. Amer. Math. Monthly 109 (3), (2002), 217-234.

[5] Y. PAN and M. WANG, On the monotonicity of positive invariant harmonic functions in the unit ball, submitted. Online at arXiv, pdf: http://arxiv.org/pdf/math.CA/0702064, other format: http://arxiv.org/format/math.CA/0702064.

[6] Ch. Pommerenke, Univalent functions, Vandenhoeck and Ruprecht, Göttingen (1975).

Yifei Pan, Department of Mathematical Sciences, Indiana University - Purdue University Fort Wayne, Fort Wayne, IN 46805-1499

School of Mathematics and Informatics, Jiangxi Normal University, Nanchang, ChInA

E-mail address: pan@ipfw.edu

Mei Wang, Department of Statistics, University of Chicago, Chicago, IL 60637

E-mail address: meiwang@galton.uchicago.edu 